\title{
INSAR DISPLACEMENT TIME SERIES MINING: A MACHINE LEARNING APPROACH
}

\author{
Homa Ansari ${ }^{1,4}$, Marc Rußwurm $^{2}$, Mohsin Ali $^{1,4}$, Sina Montazeri ${ }^{1,4}$, Alessandro Parizzi ${ }^{3}$, Xiao Xiang Zhu ${ }^{1,4}$
}

(1) Department of Earth Observation Data Science, Remote Sensing Technology Institute, German Aerospace Center (DLR)

(2) Chair of Remote Sensing Technology, Technical University of Munich (TUM)

(3) Department of SAR Signal Processing, Remote Sensing Technology Institute, German Aerospace Center (DLR)

(4) Chair of Data Science in Earth Observation, Technical University of Munich (TUM)

\begin{abstract}
Interferometric Synthetic Aperture Radar (InSAR)-derived surface displacement time series enable a wide range of applications from urban structural monitoring to geohazard assessment. With systematic data acquisitions becoming the new norm for SAR missions, millions of time series are continuously generated. Machine Learning provides a framework for the efficient mining of such big data. Here, we focus on unsupervised mining of the data via clustering the similar temporal patterns and data-driven displacement signal reconstruction from the InSAR time series. We propose a deep Long Short Term Memory (LSTM) autoencoder model which can exploit temporal relations in contrast to the commonly used shallow learning methods, such as Uniform Manifold Approximation and Projection (UMAP). We also modify the loss function to allow the quantification of uncertainties in the time series data. The two approaches are applied to the Lazufre Volcanic Complex located at the central volcanic zone of the Andes and thereby compared.
\end{abstract}

Index Terms - InSAR, Time Series, Unsupervised Learning, Latent Representation Learning, Autoencoders, Sequence Models, Deep Learning, Clustering

\section{INTRODUCTION}

Thanks to the systematic data acquisition of Sentinel-1 mission, nation-wide Earth surface displacement monitoring has become a common application of Interferometric Synthetic Aperture Radar (InSAR). This monitoring capability provides an unprecedented wealth of data, whose mining poses a challenge whilst providing a unique opportunity.

This study is part of the TecVolSA project and partially funded by the Helmholtz Association of German Research Centers. The authors would like to thank Prof. Dr. T. Walter and R. Mania from German Research Center for Geosciences (GFZ), for their insights regarding the volcanic complex, and Dr. R. Shau and Dr. F. De Zan from DLR for the provision of InSAR data.
Standard product of InSAR techniques include point-wise measurements of the earth surface; the temporal evolution of the surface elevation form displacement Time Series (TS) per point. With systematic large-scale mapping, millions of point-wise TS are at our disposal for information extraction. The sheer volume of the data as well as the diversity of the displacement patterns call for efficient data mining approaches.

The common approach in InSAR TS analysis is to retrieve the temporal displacement signal by assumng a priori models, such as linear, sinosoidal or their superimposition, followed by statistical model selection (see e.g. [1]). Although powerful, this approach does not scale well to the Big Data at hand. Moreover, the assumed displacement models may be too restrictive for detection of unknown temporal patterns. The precision of this modeling is further compromised if the implicit assumption of trend-stationarity does not hold for the displacement signals.

As an alternative solution, in this work we employ a Machine Learning (ML) approach, where the displacement models are adaptively learned from the TS rather than being restricted to a set of a priori known base functions. We adapt a general pipeline from exploratory data analysis for unsupervised mining of the TS. The clustering of similar displacement patterns and retrieving the noise-removed displacement signal are the outcome of the pipeline.

Two shallow and deep learning approaches are integrated and compared in the ML pipeline. A variation of the shallow approach has been previously used by [2] and [3]. Building on the latter works, by proposing a different shallow algorithm, we further explore the capabilities and flexibility that a deep learning approach provides for TS mining. The deep approach is based on the Long Short Term Memory (LSTM) Autoencoder (AE). In comparison to the shallow alternative, this deep learning approach allows for:

- exploitation of temporal correlations in feature extraction from the TS;

- modification of the loss function to model the data uncertainties [4] in the TS;

- enabling applications such as signal reconstruction from noisy TS and anomaly detection. 


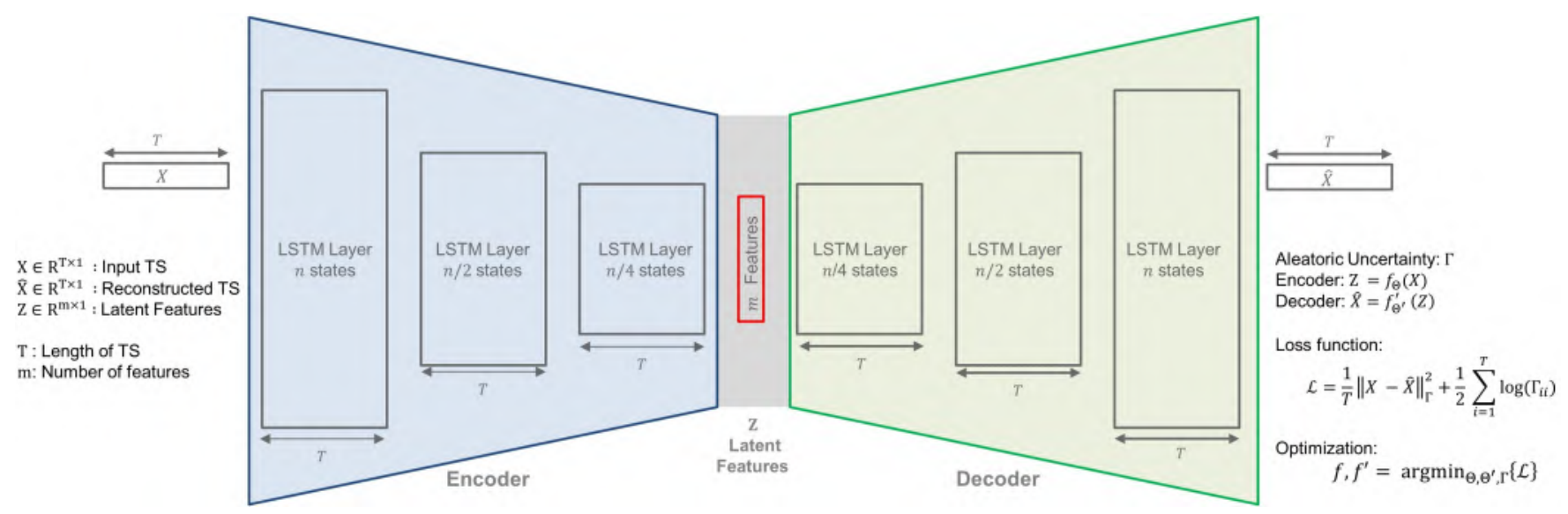

Fig. 1: The proposed Noise-Aware LSTM Autoencoder network as a deep learning approach for feature extraction from InSAR time series. The data uncertainties, shown by the diagonal matrix $\Gamma$, account for the possible residual noise in the time series.

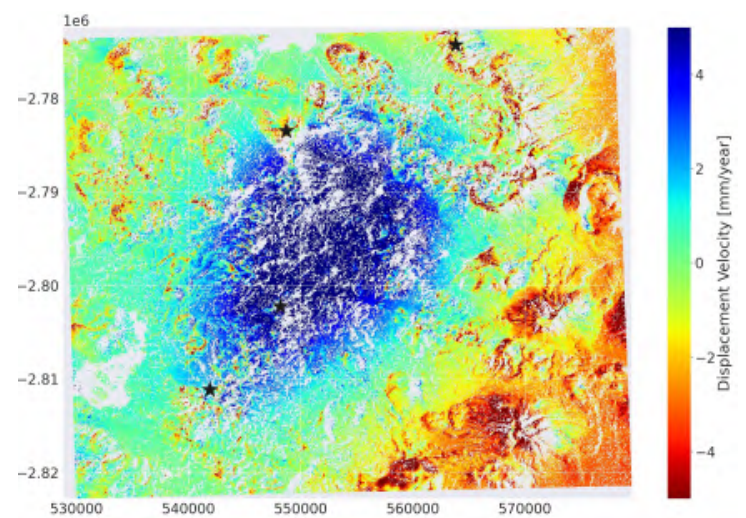

Fig. 2: Displacement velocity map of Lazufre volcanic complex, as a standard product of InSAR processing. The stars indicate the location of four volcanoes in the complex.

\section{ML PIPELINE FOR TIME SERIES MINING}

The rationale of the ML pipeline follows from common exploratory data analysis techniques. It consists of two core steps: 1) extracting informative features from the TS, thereby reducing its dimensionality; and 2) clustering similar TSs based on the retrieved features. For performing each step an arsenal of ML algorithms are at our disposal. The efficacy of this data mining approach is governed primarily by the choice of these ML algorithms and secondly by tuning their hyperparameter sets. In the following we expand on our choice of algorithms.

\subsection{Feature Extraction from Time Series}

The point-wise TS are the input of this stage. Note that, the spatial structure, i.e. potenital similarities among the TS of the neighboring points, are not exploited by the ML algorithm. Following this stage, the TS is transformed and re- duced to $m$ number of informative features. For this transformation, we choose and compare two shallow and deep learning approaches to compress the TS.

\subsubsection{Shallow learning approach}

The state-of-the-art shallow approach in dimensionality reduction is provided by the Uniform Manifold Approximation and Projection (UMAP) algorithm [5]. Exploring the topology, UMAP allows for capturing the global and local patterns in data simultaneously; it scales gracefully with the dimensionality of data, i.e. the length of TS, as well as the size of the target feature space $m$. The latter is a hyperparameter to be tuned. Despite its advantages, UMAP does not allow for exploring the temporal relations of the input data and treat the different epochs in the TS as independent and identically distributed (i.i.d.) samples.

\subsubsection{Deep learning approach}

Autoencoders (AEs) are designated neural network architectures for dimensionality reduction. The data compression in AEs is learned adaptively via a cascade of neural layers, which firstly encode the input data into a latent low dimensional feature space; and further reconstruct a version of the input data from the encoded features, via a cascade of decoding layers. The mappings in encoder and decoder are learned by minimizing a regression loss between input data and its reconstruction. We design an $\mathrm{AE}$ which exploits the temporal patterns and dependencies in the input signal by using LSTM [6] layers in the encoder and decoder. Furthermore, we modified the reconstruction loss to model the data uncertainties in the Time Series (TS) [4]. This uncertainty represents the residual atmospheric and decorrelation noise in the InSAR time series and therefore accommodates a more effective signal reconstruction and feature extraction. Figure 1 depicts the designed noise-aware LSTM-AE, while summarizing the 

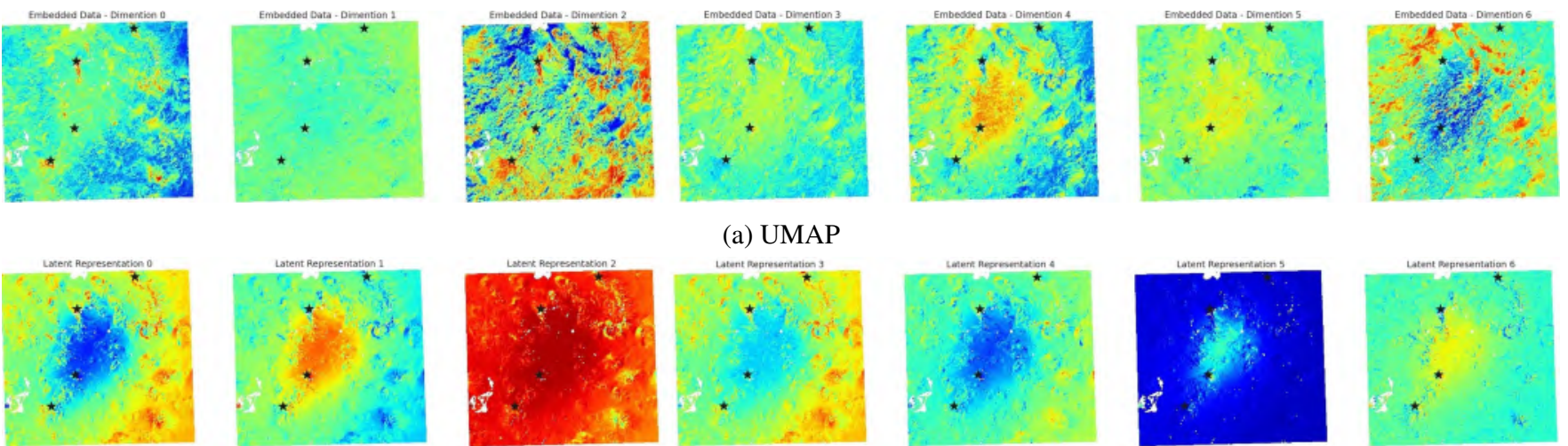

(a) UMAP
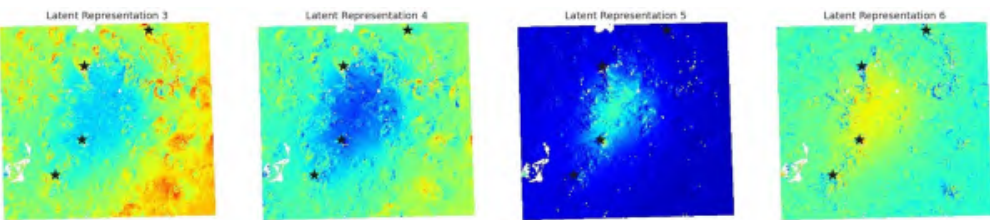

(b) Noise-Aware LSTM Autoencoder

Fig. 3: The latent representations of the displacement TS shown for (a) the shallow and (b) deep learning approaches. The stars indicate the volcanoes. A high correlation between these representations and the InSAR-derived veloctiy and elevation maps is observed (cf. Fig. 2). Further interpretation and comparisosn of the latent sources will follow in the oral presentation.
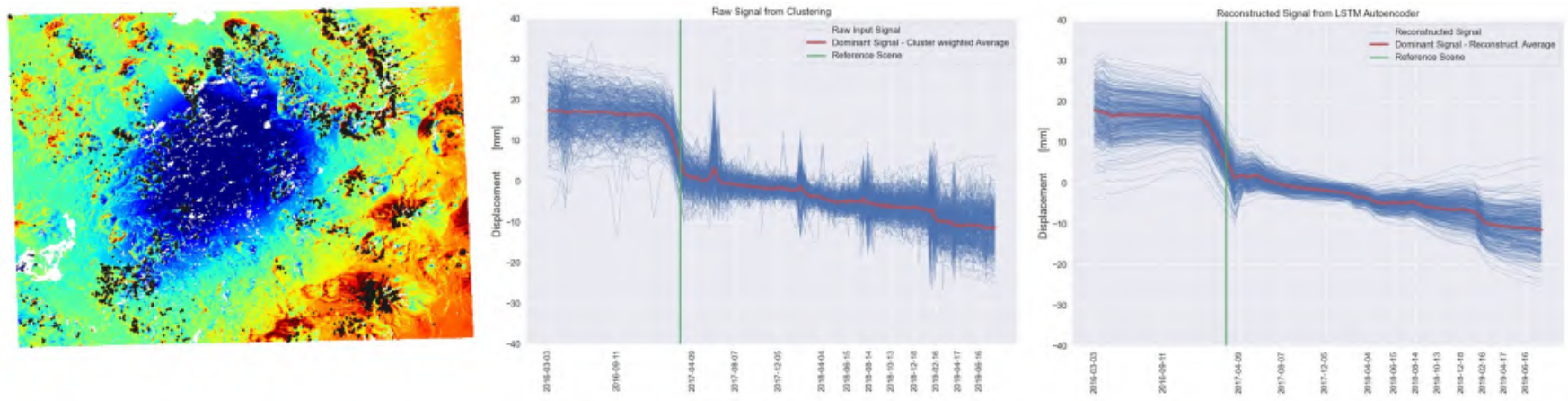

Fig. 4: An example of clustered TS for one cluster. (left) spatial distribution of the clustered samples shown by black dots overlaid on the InSAR velocity map (middle) displacement time series of the clustered samples per acquisition date, (right) the reconstructed signal of the same samples by the LSTM-AE. The selected cluster pertains to areas which undergo slope-induced displacement.

involved mappings, loss function and optimization of the network.

\subsection{Clustering based on the TS features}

Our choice of clustering algorithm is the Hierarchical Densitybased Spatial Clustering of Applications with Noise (HDBSCAN) [7]. As the name suggest, this algorithm benefits from the advantages of both hierarchical and density-based techniques, resulting in a highly automated and powerful method. Some of its decisive characteristics for the task at hand are: the possibility of soft clustering, exclusion of outlying samples, and automatic selection of important hyperparameters for clustering, e.g. distance measure, for the selection of nearby inlying samples, as well as the number of clusters.

The input of this step are the leanrned $m$ feature from the TS. Following this steps, similar point-wise measurements are clustered. Is the feature extraction and clustering effective, the clustered measurements will show a similar temporal patterns in their initial TS.

\section{APPLICATION TO LAZUFRE VOLCANIC COMPLEX}

The Lazufre volcanic complex is chosen here to demonstrate the application of the proposed TS mining approach. The site is located in central volcanic zone of the Andes and is comprised of four volcanoes spanning an area of $2500 \mathrm{~km}^{2}$. A variety of different geophysical signals are expected in the region.

\subsection{InSAR Time Series Data}

A time series of 128 Sentinel-1 acquisitions from 2016 to 2019 comprise the data set. A combination of persistent and distributed scatterers are exploited in the Interferometric Wide Area Processing (IWAP) chain [8] to process the time series. IWAP provides a unique capability in retrieving high-precision displacement time series. Compared to the state-of-the-art InSAR time series techniques, IWAP precision is especially enhanced by atmospheric corrections [9] 
and precise phase estimation for distributed scatterers [10]. The accuracy and precision of IWAP is scrutinized in [11] and [12]. Figure 2 shows the estimated displacement velocity as a standard product of InSAR and IWAP. Note that in the retrieval of the displacement TS, IWAP considers a spatial estimation and removal of the residual atmospheric signals. Using this technique, non-linear deformation signal may leak to the atmospheric estimation and thereby be removed from the exploited TS data.

\subsection{Latent Representation Learning}

Extracting features using either of the shallow or deep learning methods of 2.1 allows the representation of the TS from a 128 dimensional space into a low dimentional latent space. The dimensionality of the latent space is chosen from the range of 5 to 12 based on reconstruction performance on a separate validation dataset.

In the following, we expand on our method for vitalization of such learned latent representations. Having the displacement TS as the input signals, the algorithm outputs $m$ features per TS. Each TS is tagged by geographic coordinates. Although this spatial information is not exploited in the feature extraction, we use it to visualize the latent representations, by plotting each feature according to the coordinates of the input signal. Such visualization allows the interpretation and inspection of the latent representations. Figure 3 shows the outcome of this visualization for both UMAP and LSTM-AE.

\subsection{Time Series Clustering}

Given the latent features, the similar signals are clustered according to Section 2.2. To inspect the result, the input TS per cluster is visualized (Fig. 4 middle), the corresponding geographical coordinates of each sample TS in the cluster is provided as an overlay of points on the velocity map (Fig. 4 right). The latter allows the inspection of spatial distribution of the samples. Figure 4 shows an example of a clustered signal using the UMAP+HDBSCAN pipeline.

\subsection{Further capabilities of the LSTM Autoencoder}

Aside from the clustering application, the employed LSTMAE allows for the reconstruction of the displacement signal from the noisy input TS. Figure 4 right depicts an example of such signal recovery. Moreover, the introduction of data uncertainty in the optimization of the network provides a mean to quantify the noise in the TS and take this noise into account in the reconstruction of the displacement signal.

\section{SUMMARY AND OUTLOOK}

In this short abstract we summarized two deep and shallow learning approaches for efficient InSAR TS mining. For the sake of brevity, in depth comparison of the two frameworks as well as their merits in latent representation learning and clustering will be addressed in the oral presentation.

A relevant future research direction is the transferability of such TS mining approaches; i.e. the applicability of a learned model based on data sets used in thr training process to a data set from a different region of interest. This transferebility is in particular challenging for InSAR data; reasons are: the specificity of InSAR signal and error sources to the signal propagation medium and surface characteristic; such as topography, land-cover etc., as well as the sensor geometry.

\section{REFERENCES}

[1] Ling Chang and Ramon F. Hanssen, "A probabilistic approach for InSAR time-series postprocessing," IEEE Transactions on Geoscience and Remote Sensing, 2015.

[2] Matthew Gaddes, Andy Hooper, and Marco Bagnardi, "Using machine learning to automatically detect volcanic unrest in a time series of interferograms," Journal of Geophysical Research: Solid Earth.

[3] Bas van de Kerkhof, Victor Pankratius, Ling Chang, Rob van Swol, and Ramon F Hanssen, "Individual scatterer model learning for satellite interferometry," IEEE transactions on geoscience and remote sensing.

[4] Alex Kendall and Yarin Gal, "What uncertainties do we need in bayesian deep learning for computer vision?," in Advances in neural information processing systems.

[5] Leland McInnes, John Healy, Nathaniel Saul, and Lukas Großberger, "UMAP: Uniform manifold approximation and projection," Journal of Open Source Software.

[6] Sepp Hochreiter and Jürgen Schmidhuber, "Long short-term memory," Neural computation.

[7] Ricardo JGB Campello, Davoud Moulavi, and Jörg Sander, "Density-based clustering based on hierarchical density estimates," in Pacific-Asia conference on knowledge discovery and data mining.

[8] Nico Adam, Fernando Rodriguez Gonzalez, Alessandro Parizzi, and Ramon Brcic, "Wide area Persistent Scatterer Interferometry: Current developments, algorithms and examples," in IEEE Geoscience and Remote Sensing Symposium.

[9] Xiaoying Cong, Ulrich Balss, Fernando Rodriguez Gonzalez, and Michael Eineder, "Mitigation of tropospheric delay in sar and insar using nwp data: Its validation and application examples," Remote Sensing.

[10] Homa Ansari, Francesco De Zan, and Richard Bamler, "Efficient Phase Estimation for Interferogram Stacks," IEEE Transactions on Geoscience and Remote Sensing.

[11] Homa Ansari, Alessandro Parizzi, and Francesco De Zan, "Study of systematic bias in measuring surface deformation with SAR interferometry," IEEE Transactions on Geoscience and Remote Sensing, 2020.

[12] Alessandro Parizzi, Ramon Brcic, and Francesco De Zan, "InSAR performance for large-scale deformation measurement," IEEE Transactions on Geoscience and Remote Sensing, 2020. 